\title{
Electromagnetic Modeling of Organic Light-Emitting Devices
}

\author{
Hung-Chi Chen, Jiun-Haw Lee, Member, IEEE, Chia-Chiang Shiau, \\ Chih-Chung Yang, Senior Member, IEEE, and Yean-Woei Kiang, Member, IEEE
}

\begin{abstract}
Based on the rigorous electromagnetic wave theory, a numerical model for simulating the radiation characteristics of organic light-emitting devices (OLEDs) is developed. In particular, a novel method for overcoming the numerical difficulty in taking the thick glass substrate into account is proposed. The numerical results confirm the importance of the effects of the thick glass substrate. The algorithms based on the numerical model are then used for evaluating the dependencies of OLED radiation characteristics on various parameters, including the thickness of different device layers and the cathode metal variety. In the study of the effect of emission layer (EML) thickness, it is found that the radiation spectral peak red shifts with increasing EML thickness. This trend is consistent with the experimental result.
\end{abstract}

Index Terms-Organic light-emitting device (OLED), simulation.

\section{INTRODUCTION}

$\mathbf{O}$ RGANIC light-emitting devices (OLEDs) have attracted much attention because of their advantages such as lowpower consumption, high brightness, high contrast, and low cost [1], [2]. Conventional OLEDs are fabricated on glass substrates of several hundred micrometers in thickness. In a typical device, an indium-tin-oxide (ITO) anode, several organic layers, and a metal cathode are deposited on the glass substrate. The thickness of each layer is in the range of several tens of nanometers. Electroluminescence (EL) is generated from the organic layers and coupled out of the device from the ITO side. Because the total thickness of the organic layers is comparable to the wavelength, the reflections at various interfaces lead to significant interference effects. By varying the thickness of the organic layers, the external quantum efficiency, output spectrum, and viewing angle are significantly changed [3], [4]. To understand the optical characteristics of an OLED, a simulation of the EL wave propagation in such a device is very important [5]. Although the scattering and transmission matrix formulations can be used to successfully simulate the

Manuscript received August 10, 2005; revised February 14, 2006. This work was supported by the National Science Council, R.O.C., under Grant NSC 92-2215-E-002-025 and by Vitex Systems Inc.

H.-C. Chen is with the Department of Electrical Engineering, National Taiwan University, Taipei 10617, Taiwan, R.O.C. (e-mail: rockychen@cc.ee. ntu.edu.tw)

J.-H. Lee is with the Graduate Institute of Electro-Optical Engineering and the Department of Electrical Engineering, National Taiwan University, Taipei 10617, Taiwan, R.O.C. (e-mail: jhlee@cc.ee.ntu.tw).

C.-C. Shiau and C.-C. Yang are with the Graduate Institute of ElectroOptical Engineering, National Taiwan University, Taipei 10617, Taiwan, R.O.C. (e-mail: r92941057@ntu.edu.tw; ccy@cc.ee.ntu.tw).

Y.-W. Kiang is with the Department of Electrical Engineering and the Graduate Institute of Communication Engineering, National Taiwan University, Taipei 10617, Taiwan R.O.C. (e-mail:ywkiang@ntu.edu.tw).

Digital Object Identifier 10.1109/JLT.2006.874591

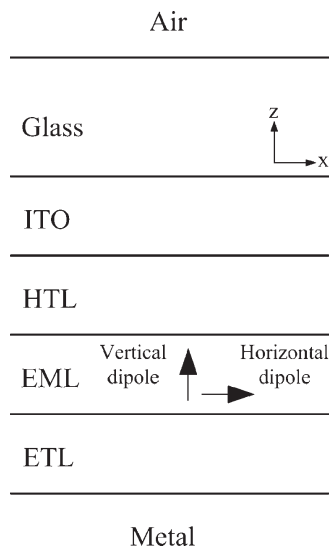

Fig. 1. Schematic illustration of a typical OLED structure.

light propagation properties of organic photovoltaic devices [6], [7], they cannot be directly applied to OLEDs because an OLED uses a thick glass substrate, which results in computational difficulty. In addition, although in the top-emission OLED configuration, the simulation results could be easily obtained without considering the thick glass substrate [8], [9], the effect of the thick glass substrate needs to be considered in a bottom-emission OLED. Electromagnetic wave propagation in stratified media has long been an important classical problem [10], [11]; however, it is still a difficult task to rigorously evaluate the radiation in a bottom-emission device. Recently, radiation from a dipole embedded in a dielectric slab was analytically treated [12]. In this treatment, both radiated and guided powers were investigated.

In this research, we develop a rigorous and efficient numerical method to simulate the radiation characteristics of an OLED. In particular, we intend to overcome the numerical difficulty due to the thick glass layer. To reach this goal, we first neglect the air region, and assume a semi-infinitely thick glass layer. In this situation, the wave field distribution in the glass layer can be computed using the saddle-point method [13]. Then, the air region is taken into account in the practical device design. An incident wave is multiply reflected and transmitted into the air region to give the output radiation. The obtained field distribution in the glass layer with the last assumption is used for the whole device simulation. Because the development of our numerical algorithms is based on the general electromagnetic theory, the proposed method is, in principle, applicable to an OLED consisting of any number of layers. For a dipole source operating at a single frequency, strong interference of the coherent fields reflected from interfaces between layers results in a rapidly oscillatory radiation pattern. However, in 
TABLE I

OLED PARAMETERS USED FOR SIMULATION

\begin{tabular}{|l|l|l|l|}
\hline Parameter & Symbol & Value & Unit \\
\hline Refractive index of the air region & $n_{1}$ & 1 & \\
\hline Refractive index of the glass layer & $n_{2}$ & 1.48 & \\
\hline Refractive index of the ITO (indium-tin oxide) layer & $n_{3}$ & 1.8 & \\
\hline Refractive index of the HTL (hole-transport layer) & $n_{4}$ & 1.7 & \\
\hline Refractive index of the EML (emission layer) & $n_{5}$ & 1.8 & \\
\hline Refractive index of the ETL (electron-transport layer) & $n_{6}$ & 1.7 & \\
\hline Refractive index of the metal cathode & $n_{\text {Metal }}$ & $\sqrt{\varepsilon_{r_{M}}}$ & \\
\hline Position of dipole sources & $z_{0}$ & 0.23 & $\mu \mathrm{m}$ \\
\hline Lower boundary of the ETL & $z_{6}$ & 0 & $\mu \mathrm{m}$ \\
\hline Lower boundary of the EML & $z_{5}$ & 0.2 & $\mu \mathrm{m}$ \\
\hline Lower boundary of the HTL & $z_{4}$ & 0.23 & $\mu \mathrm{m}$ \\
\hline Lower boundary of the ITO layer & $z_{3}$ & 0.39 & $\mu \mathrm{m}$ \\
\hline Lower boundary of the glass layer & $z_{2}$ & 0.475 & $\mu \mathrm{m}$ \\
\hline Lower boundary of the air region & 850.475 & $\mu \mathrm{m}$ \\
\hline
\end{tabular}

reality, light emission from a dipole source should have a finite bandwidth. Hence, the interference effect will be smoothened. In our calculations, the resultant radiation field is obtained by coherently averaging the fields over a spectral range. In this paper, the theoretical formulations are given in Section II. The numerical results and related discussions are presented in Section III. Finally, conclusions are drawn in Section IV.

\section{THEORETICAL Formulations}

Consider an OLED with a seven-layer structure, as schematically shown in Fig. 1. The device parameters are listed in Table I. Although our theoretical model is applicable to any multilayer structure, the following derivations are based on a seven-layer device. We first solve the governing equation for the electric Hertzian vector potential $\vec{\Pi}$, which is due to a vertical or horizontal dipole embedded in the emission layer (EML) and near the upper boundary of the layer. Then, with the $e^{j \omega t}$ timeharmonic dependence, the electric and magnetic fields can be evaluated through the following expressions [13]:

$$
\begin{aligned}
& \vec{E}=\nabla(\nabla \cdot \vec{\Pi})+k_{0}^{2} n^{2} \vec{\Pi} \\
& \vec{H}=j \omega \varepsilon_{0} n^{2} \nabla \times \vec{\Pi} .
\end{aligned}
$$

Here, $n$ is the complex refractive index ( $n=n_{i}$ in the $i$ th layer) and $k_{0}=\omega \sqrt{\mu_{0} \varepsilon_{0}}=2 \pi / \lambda$ is the wave number or propagation constant in vacuum.

The equation governing $\vec{\Pi}$ can be derived as

$$
\left(\nabla^{2}+k_{0}^{2} n_{5}^{2}\right) \vec{\Pi}(\vec{r})=\frac{-\vec{p} \delta\left(\vec{r}-\vec{r}^{\prime}\right)}{\varepsilon_{0} n_{5}^{2}}
$$

in the fifth EML, and

$$
\left(\nabla^{2}+k_{0}^{2} n_{i}^{2}\right) \vec{\Pi}(\vec{r})=0
$$

in the $i$ th nonemission layer. Here, $\vec{p}$ denotes a dipole moment located at $\vec{r}^{\prime}$. We employ the property of axial symmetry and take the Fourier-Bessel transform to obtain an integral expression for the Hertzian vector potential in each layer. The boundary conditions include the continuity of the tangential electric and magnetic fields across each boundary. Using the boundary conditions at each interface, we can determine the unknown coefficients in the integral expression of $\vec{\Pi}$. With this procedure, all the field components can be evaluated through (1).

To be specific, we first consider a vertical dipole source with its moment $\vec{p}=\hat{z} p$ located at $\rho=0$ and $z=z_{0}$ in the fifth EML. After some mathematical manipulations, the Hertzian vector potential $\vec{\Pi}_{i}$ in the $i$ th region can be derived as

$$
\vec{\Pi}_{1}(\rho, z)=\hat{z} \frac{j p}{4 \pi \varepsilon_{0} n_{5}^{2}} \int_{0}^{\infty} T_{1 b}(\alpha) J_{0}(\alpha \rho) e^{-j \tau_{1}\left(z-z_{1}\right)} \alpha d \alpha
$$

in the air region

$$
\begin{aligned}
\vec{\Pi}_{i}(\rho, z)=\hat{z} \frac{j p}{4 \pi \varepsilon_{0} n_{5}^{2}} \int_{0}^{\infty} & {\left[T_{i a}(\alpha) e^{j \tau_{i}\left(z-z_{i}\right)}\right.} \\
& \left.+T_{i b}(\alpha) e^{-j \tau_{i}\left(z-z_{i}\right)}\right] J_{0}(\alpha \rho) \alpha d \alpha
\end{aligned}
$$


in the $i$ th $(i=2,3,4,6)$ layer

$$
\begin{aligned}
& \vec{\Pi}_{5}(\rho, z)=\hat{z} \frac{j p}{4 \pi \varepsilon_{0} n_{5}^{2}} \int_{0}^{\infty}\left[T_{5 a}(\alpha) e^{j \tau_{5}\left(z-z_{5}\right)}\right. \\
& \left.\quad+T_{5 b}(\alpha) e^{-j \tau_{5}\left(z-z_{5}\right)}-\frac{1}{\tau_{5}} e^{-j \tau_{5}\left|z-z_{0}\right|}\right] J_{0}(\alpha \rho) \alpha d \alpha
\end{aligned}
$$

in the fifth EML, and

$$
\vec{\Pi}_{7}(\rho, z)=\hat{z} \frac{j p}{4 \pi \varepsilon_{0} n_{5}^{2}} \int_{0}^{\infty} T_{7 a}(\alpha) J_{0}(\alpha \rho) e^{j \tau_{7}\left(z-z_{6}\right)} \alpha d \alpha
$$

in the metal layer. Here, $J_{0}$ is the first-kind Bessel function of zeroth order, $T_{i a}(\alpha)$ and $T_{i b}(\alpha)$ are the expansion coefficients to be determined, $\tau_{i}=\left(k_{0}^{2} n_{i}^{2}-\alpha^{2}\right)^{1 / 2}$, and $z_{i}$ is the $z$-coordinate of the lower boundary in the $i$ th layer. Note that only the outgoing waves exist in the air and the cathode regions.

By using the boundary conditions for the continuities of the tangential electric and magnetic fields across all the boundaries between different layers, we can derive the algebraic equations for the expansion coefficients $T_{i a}(\alpha)$ and $T_{i b}(\alpha)$. After solving these equations, the Hertzian vector potential and hence the electric and magnetic fields in all regions can then be calculated accordingly.

Now, we consider the radiation characteristics from a horizontal dipole with the dipole moment $\vec{p}=\hat{x} p$ located at $\rho=0$ and $z=z_{0}$ in the EML. We again employ the Hertzian vector potential $\vec{\Pi}\left(=\hat{x} \Pi_{x}+\hat{z} \Pi_{z}\right)$, which satisfies the Helmholtz (2) and (3). Note that both the $x$ - and $z$-components of the Hertzian vector potential are needed for all the electromagnetic fields to satisfy Maxwell's equations and the boundary conditions [13].

Using the derivation procedures similar to the case of a vertical dipole, we can derive the representation of $\vec{\Pi}_{i}(\rho, \phi, z)$ in the $i$ th region as

$$
\begin{aligned}
\vec{\Pi}_{1}(\rho, \phi, z) & =\hat{x} \frac{j p}{4 \pi \varepsilon_{0} n_{5}^{2}} \int_{0}^{\infty} U_{1 b}(\alpha) J_{0}(\alpha \rho) e^{-j \tau_{1}\left(z-z_{1}\right)} \alpha d \alpha \\
& +\hat{z} \frac{j p \cos \phi}{4 \pi \varepsilon_{0} n_{5}^{2}} \int_{0}^{\infty} A_{1 b}(\alpha) J_{1}(\alpha \rho) e^{-j \tau_{1}\left(z-z_{1}\right)} \alpha^{2} d \alpha
\end{aligned}
$$

in the air region

$$
\begin{aligned}
& \vec{\Pi}_{i}(\rho, \phi, z) \\
& =\hat{x} \frac{j p}{4 \pi \varepsilon_{0} n_{5}^{2}} \int_{0}^{\infty}\left[U_{i a}(\alpha) e^{j \tau_{i}\left(z-z_{i}\right)}+U_{i b}(\alpha) e^{-j \tau_{i}\left(z-z_{i}\right)}\right] \\
& \times J_{0}(\alpha \rho) \alpha d \alpha+\hat{z} \frac{j p \cos \phi}{4 \pi \varepsilon_{0} n_{5}^{2}} \\
& \quad \times \int_{0}^{\infty}\left[A_{i a}(\alpha) e^{j \tau_{i}\left(z-z_{i}\right)}+A_{i b}(\alpha) e^{-j \tau_{i}\left(z-z_{i}\right)}\right] J_{1}(\alpha \rho) \alpha^{2} d \alpha
\end{aligned}
$$

in the $i$ th $(i=2,3,4,6)$ layer

$$
\begin{aligned}
& \vec{\Pi}_{5}(\rho, \phi, z) \\
& =\hat{x} \frac{j p}{4 \pi \varepsilon_{0} n_{5}^{2}} \int_{0}^{\infty}\left[U_{5 a}(\alpha) e^{j \tau_{5}\left(z-z_{5}\right)}+U_{5 b}(\alpha) e^{-j \tau_{5}\left(z-z_{5}\right)}\right. \\
& \left.\quad-\frac{1}{\tau_{5}} e^{-j \tau_{5}\left|z-z_{0}\right|}\right] J_{0}(\alpha \rho) \alpha d \alpha+\hat{z} \frac{j p \cos \phi}{4 \pi \varepsilon_{0} n_{5}^{2}} \\
& \quad \times \int_{0}^{\infty}\left[A_{5 a}(\alpha) e^{j \tau_{5}\left(z-z_{5}\right)}+A_{5 b}(\alpha) e^{-j \tau_{5}\left(z-z_{5}\right)}\right] J_{1}(\alpha \rho) \alpha^{2} d \alpha
\end{aligned}
$$

in the fifth EML, and

$$
\begin{array}{r}
\vec{\Pi}_{7}(\rho, \phi, z)=\hat{x} \frac{j p}{4 \pi \varepsilon_{0} n_{5}^{2}} \int_{0}^{\infty} U_{7 a}(\alpha) J_{0}(\alpha \rho) e^{j \tau_{7}\left(z-z_{6}\right)} \alpha d \alpha \\
+\hat{z} \frac{j p \cos \phi}{4 \pi \varepsilon_{0} n_{5}^{2}} \int_{0}^{\infty} A_{7 a}(\alpha) J_{1}(\alpha \rho) e^{j \tau_{7}\left(z-z_{6}\right)} \alpha^{2} d \alpha
\end{array}
$$

in the metal layer. Here, the dependence of the azimuth angle $\phi$ is explicitly presented. The expansion coefficients $U_{i a}(\alpha)$, $U_{i b}(\alpha), A_{i a}(\alpha)$, and $A_{i b}(\alpha)$ can be obtained by solving the simultaneous algebraic equations derived from the boundary conditions.

In computing the radiation fields in the air region, we employ the method of saddle point for integrating with respect to $\alpha$. However, because the thickness of the glass layer is usually much larger than the wavelength (by several hundred times), we cannot separate the slowly varying and the fast-varying terms in the expressions of $T_{i a}(\alpha), T_{i b}(\alpha), U_{i a}(\alpha), U_{i b}(\alpha), A_{i a}(\alpha)$, and $A_{i b}(\alpha)$ in using the saddle-point method. To overcome this difficulty, we first assume that the glass layer is semi-infinitely thick. With the new six-layer structure, we employ the saddlepoint method to determine the mathematical expression for the radiation field $E_{\text {glass }}(\rho, z)$ at the original interface between the air region and the glass layer. Based on this idea, the following formula for the saddle-point method can be readily used:

$$
\begin{gathered}
\int_{-\infty}^{\infty} f(\alpha) e^{-j \alpha \rho-j \tau_{2}\left(z-z_{2}\right)} d \alpha \approx f\left(k_{0} n_{2} \sin \theta_{\text {glass }}\right) \sqrt{\frac{2 \pi}{k_{0} n_{2} R_{2}}} \\
\cdot k_{0} n_{2} \cos \theta_{\text {glass }} \cdot e^{-j k_{0} n_{2} R_{2}+j \frac{\pi}{4}}
\end{gathered}
$$

Here, $R_{2}=\left(\rho^{2}+\left(z-z_{2}\right)^{2}\right)^{1 / 2}, \theta_{\text {glass }}$ is the zenithal angle, and $(\rho, z)$ represents an observation point on the original interface between the air region and the glass layer.

After obtaining the aforementioned results, we put the air region back to the device structure. Now, the $E_{\text {glass }}(\rho, z)$ field becomes an incident field onto the glass/air interface. It will propagate in the glass layer subject to multiple reflections and transmissions, as schematically shown in Fig. 2(a) and (b) for the parallel and perpendicular polarization components, respectively. 


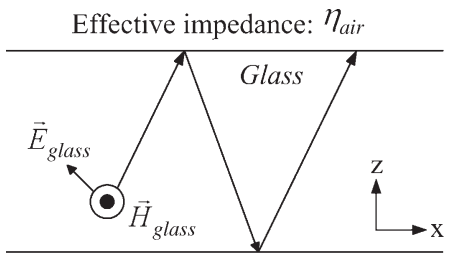

Effective impedance: $\eta_{\text {eff }}$ (parallel polarization)

(a)

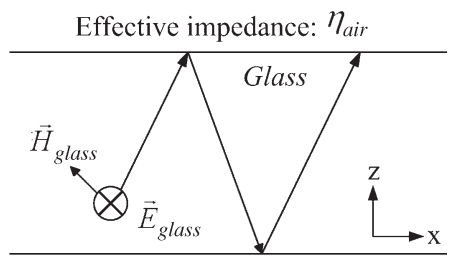

Effective impedance: $\eta_{\text {eff }}$ (perpendicular polarization)

(b)

Fig. 2. (a) Parallelly and (b) perpendicularly polarized wave multiply reflected and transmitted in the glass layer.

Employing the electromagnetic theory with matrix multiplication enables us to evaluate the effective impedance $\eta_{\text {eff }}$ of the multilayer structure at a location below the glass layer. Let us first use the Fresnel formulas to evaluate the reflection coefficient $\Gamma_{2}$ from the glass layer to the rest multilayer structure and the reflection coefficient $\Gamma_{1}$ and transmission coefficient $T_{1}$ from the glass layer to the air region. We can then determine the effective transmission coefficient $T_{\text {eff }}$ for $E_{\text {glass }}(\rho, z)$ to transmit into the air region in the form

$$
T_{\text {eff }}=\frac{T_{1}}{1-\Gamma_{1} \Gamma_{2} e^{-2 j k_{0} n_{\text {glass }} d_{\text {glass }} \sec \left(\theta_{\text {glass }}\right)}} .
$$

Here, $n_{\text {glass }}$ and $d_{\text {glass }}$ are the refractive index and the thickness of the glass layer, respectively. Note that the two polarization components (parallel and perpendicular) with different reflection $\left(\Gamma_{2}, \Gamma_{1}\right)$ and transmission $\left(T_{1}\right)$ coefficients should be treated separately.

In fact, the radiated field in the glass layer $E_{\text {glass }}(\rho, z)$ is not a plane wave but a spreading beam wave. Using the principle of energy conservation and Snell's law [14], we can derive the spreading factor as

$$
S=\left[\frac{\cos \theta_{\text {glass }}}{\cos \theta_{\text {air }}}\right]^{2} \frac{\sqrt{\cos \theta_{\text {air }}}}{\sqrt[4]{1-\left(\frac{n_{\text {air }}}{n_{\text {glass }}} \sin \theta_{\text {air }}\right)^{2}}}
$$

Here, $\theta_{\text {glass }}$ is the incident angle, and $\theta_{\text {air }}$ is the refractive angle from the glass layer to the air region. Therefore, the radiation field for each polarization due to a vertical or horizontal dipole source embedded in the EML can finally be expressed in the following form:

$$
E_{\text {air }}=E_{\text {glass }} \cdot T_{\text {eff }} \cdot S \text {. }
$$

A light wave cannot be emitted by an electric dipole of an infinitely long coherent time (i.e., of an infinitesimal bandwidth). In our calculations, we assume that a dipole source emits a spectrum of finite spectral width $\left(\lambda_{0}-\Delta \lambda, \lambda_{0}+\Delta \lambda\right)$. The spectral width is determined by the material property of an OLED. Here, we coherently average the radiated fields over the spectral range $(2 \Delta \lambda)$ of $0.5 \mathrm{~nm}$.

To simulate a real device, we consider the radiation from a set of randomly oriented dipole sources embedded in the EML. The number of dipoles used is $10^{5}$. For each dipole oriented in the direction $\left(\theta_{r}, \phi_{r}\right)$, it is decomposed into a vertical component and a horizontal component, from which the radiation can be individually calculated. Here, the random variable $\theta_{r}$ is uniformly distributed between 0 and $\pi$, and the random variable $\phi_{r}$ is uniformly distributed between 0 and $2 \pi$. We generate random samples of dipole orientation and calculate the incoherent summation of their radiation contributions to give the output of the considered OLED.

\section{NumERICAL RESUlts AND Discussions}

In this section, we demonstrate and discuss the numerical results based on the formulations in the previous section. Most simulation parameters are listed in Table I, unless specified otherwise. In Fig. 3(a) and (b), we show the radiation spectrum by varying the thickness of the glass layer from 0.5 to $850 \mu \mathrm{m}$. Here, the assumed photoluminescence (PL) spectrum of the EML layer is plotted as the dot-dashed curve. Note that the spectrum is taken in the zenithal direction, i.e., $\theta=0$ (the same condition is used hereafter). When the glass layer is thin, as shown in Fig. 3(a), the oscillation behavior becomes more prominent as the glass layer thickness increases. To interpret this trend, we can use a simple model for estimating the free spectral range $\Delta v$ of the Fabry-Pérot interference fringe as

$$
\Delta v=\frac{c}{2 n d \cdot \cos \theta_{i}}
$$

Here, $c$ is the speed of light, $n$ is the average refractive index of device materials, $d$ is the thickness of the effective microcavity, and $\theta_{i}$ is the incident angle. The free spectral ranges (in terms of wavelength) for the cases of glass thickness at $0.5,1$, and $3 \mu \mathrm{m}$ are about 156,78 , and $26 \mathrm{~nm}$ (centered at $478 \mathrm{~nm}$ ), respectively, resulting from the microcavity formed by the electron transport layer (ETL)/cathode interface and the glass/air interface. In the case of $0.5-\mu \mathrm{m}$ glass layer thickness, the free spectral range is broader than the PL spectrum such that no Fabry-Pérot modulation can be observed. In the case of $1-\mu \mathrm{m}$ glass layer thickness, the free spectral range is reduced to $78 \mathrm{~nm}$. Hence, Fabry-Pérot modulation is shown at the spectral tails. Following this trend, strong modulation is shown in the case of 3- $\mu \mathrm{m}$ glass layer thickness, as confirmed by the corresponding curve in Fig. 3(a). On the other hand, when the glass layer is thick, i.e., up to several hundred micrometers, the free spectral range is reduced to the range of tenths of a nanometer such that the Fabry-Pérot oscillation is beyond the noticeable spectral resolution. Therefore, the three curves in Fig. 3(b) are essentially smooth. The comparison between 


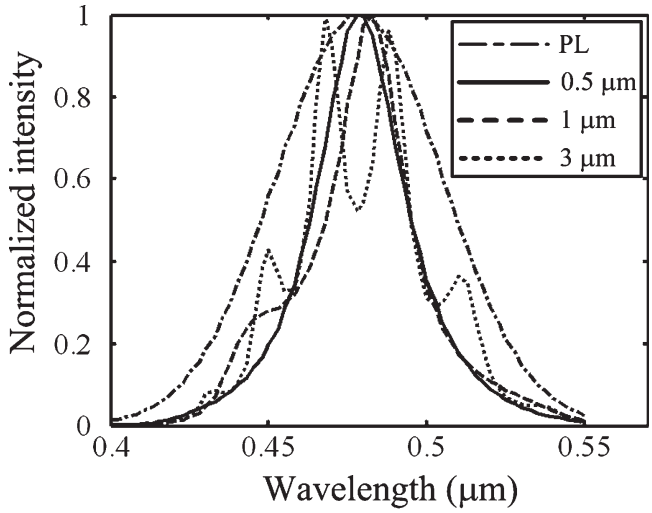

(a)

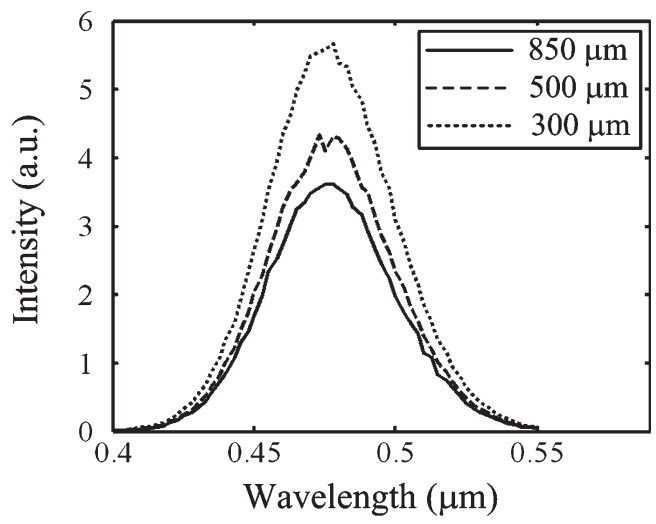

(b)

Fig. 3. Simulation results of the EL spectra at $\theta=0$ with various (a) thin and (b) thick glass layer thicknesses. The assumed PL spectrum of the EML layer is plotted as the dot-dashed curve.

Fig. 3(a) and (b) indicates the importance of our simulation model. With our accurate algorithm, one can clearly see the oscillating spectrum by using a numerical model of thin glass layer. The oscillating spectrum is not observed in a real device. It is noted that the output intensity decreases with increasing glass thickness. With a thicker glass layer, the waveguiding effect becomes stronger such that the external quantum efficiency is decreased.

Fig. 4(a) and (b) shows the viewing angle dependence of radiation intensity with different glass layer thicknesses at $\lambda=470$ nm. In Fig. 4(a), the Fabry-Pérot modulation becomes stronger as the glass thickness increases from 0.5 to $3 \mu \mathrm{m}$. This trend is consistent with the results in Fig. 3(a). In addition, the same trend in Fig. 3(b) can be observed in Fig. 4(b). Meanwhile, the radiation intensity variation trend is the same as that described later in the text. In addition, due to the weaker waveguiding effect in a device of thinner glass layer, its viewing angle is larger.

In the following paragraphs, we will use the built numerical model for investigating the radiation characteristics of an OLED by varying various parameters. First, in Fig. 5, we show the radiation spectra for several cathode materials. The complex dielectric constants of those metals were obtained from [15]. In Fig. 5, one can see that the spectral peak position varies among different cathode metals. It is interesting to note that the output spectra in the cases of $\mathrm{Au}, \mathrm{Al}$, and perfect electric conductor

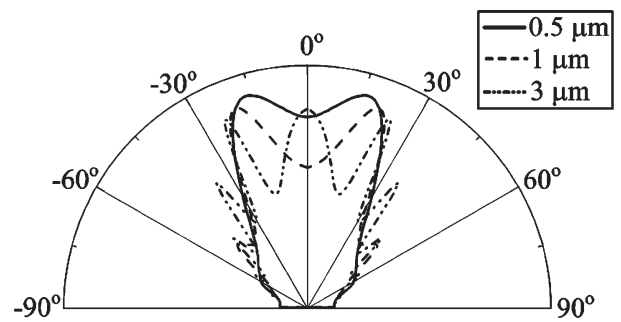

(a)

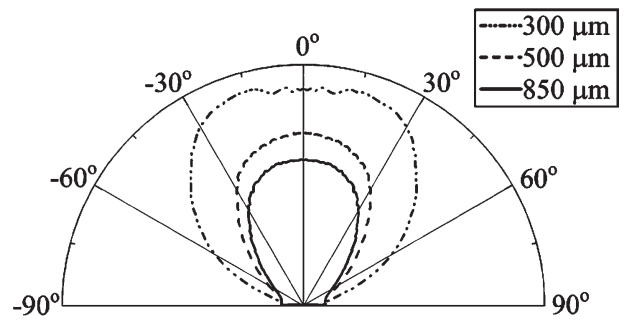

(b)

Fig. 4. Viewing angle dependence of the radiation pattern at $\lambda=470 \mathrm{~nm}$ with various (a) thin and (b) thick glass layer thicknesses.

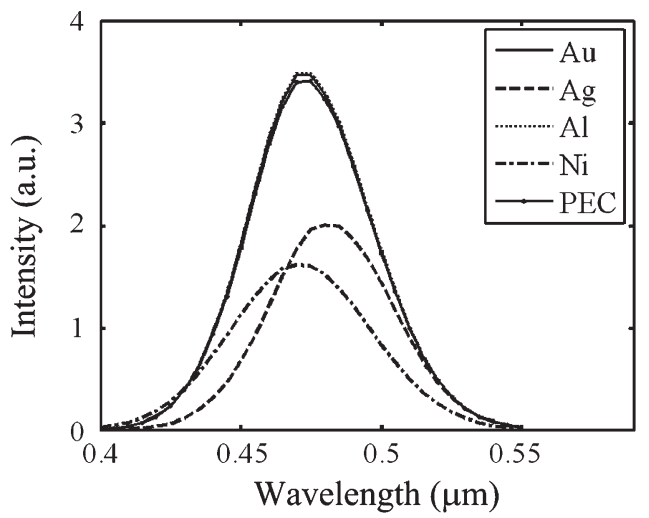

Fig. 5. Simulation results of normalized EL spectra at $\theta=0$ with various cathode metals.

(PEC) as the cathode materials are almost identical. In addition, their peak intensities are about the same and are higher than those of $\mathrm{Ag}$ and $\mathrm{Ni}$ as cathode materials. The spectral peaks are located at 470, 475, and $480 \mathrm{~nm}$ when the cathode materials are $\mathrm{Ni}, \mathrm{Au}$, and $\mathrm{Ag}$, respectively. The results shown in Fig. 5 imply that a careful selection of cathode material may help in increasing the output intensity and controlling the radiation spectrum.

Fig. 6(a) shows the normalized spectra of four devices with different EML thicknesses of 20, 30, 40, and $60 \mathrm{~nm}$. It clearly shows the red-shift trend with increasing the EML thickness. For comparison, a series of OLEDs was fabricated. We used ITO-coated glass substrates of low sheet resistivity $(10 \Omega / \mathrm{sq})$ and small surface roughness (standard deviation $<1 \mathrm{~nm}$ ). The size of the active region was $1 \times 1 \mathrm{~cm}^{2}$. In those devices, we used $\mathrm{N}, \mathrm{N}^{\prime}$-diphenyl-N, $\mathrm{N}^{\prime}$-bis(1-napthyl)-1, $1^{\prime}$-biphenyl-4, $4^{\prime}$-diamine (NPB) as the hole transport layer (HTL) material, 4,4'-bis[2-(4-(N,N-diphenylamino)phenyl)vinyl]biphenyl (DPAVBi) as the blue dopant of the EML, (9,10-bis (29-naphthyl)73 anthracene) (ADN) as the host of the EML, and tris-(8-hydroxyquinoline) aluminum (Alq3) as the ETL 


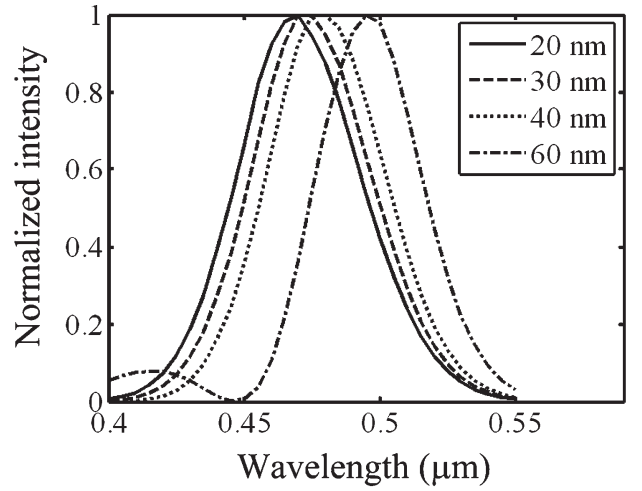

(a)

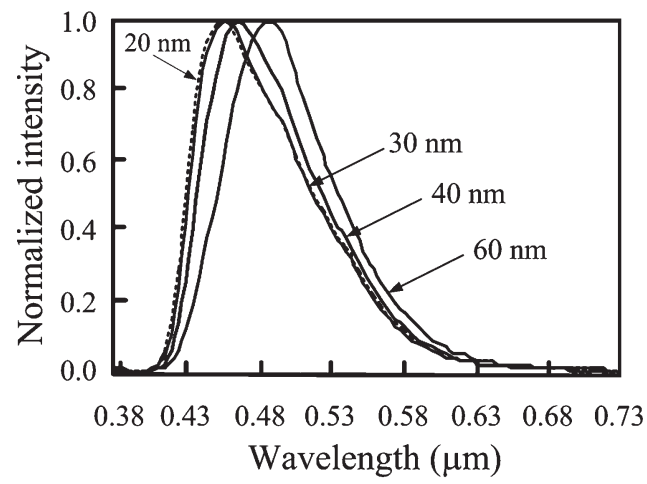

(b)

Fig. 6. (a) Simulation and (b) experimental results of the normalized EL spectra at $\theta=0$ with various EML thicknesses.

material. The doping concentration of DPAVBi in ADN was $2 \%$ in mole ratio. The thicknesses of the HTL and ETL were kept at 160 and $200 \mathrm{~nm}$, respectively. The thickness of the EML was varied at $20,30,40$, and $60 \mathrm{~nm}$. LiF of $1.2 \mathrm{~nm}$ in thickness was used between the ETL and the aluminum cathode for a more efficient electron injection. The thickness of the aluminum cathode was $100 \mathrm{~nm}$. After organic and metal deposition, the devices were encapsulated in the glove box with the $\mathrm{O}_{2}$ and $\mathrm{H}_{2} \mathrm{O}$ concentrations below $1 \mathrm{ppm}$. Devices were characterized with an SR-2 photometer for the brightness measurement. The resulting spectra are shown in Fig. 6(b). The red-shift trend with increasing layer thickness shows a similar behavior to the simulation results, as shown in Fig. 6(a).

Fig. 7 shows the spectral variation with HTL thickness. The spectral peak position blue shifts from 483 to $471 \mathrm{~nm}$ when the HTL thickness increases from 80 to $130 \mathrm{~nm}$. However, it red shifts from 471 to $483 \mathrm{~nm}$ when the thickness is further increased from 130 to $200 \mathrm{~nm}$. Typically, when the thickness of a thin film (such as ITO, HTL, and EML) changes, the light intensity of a particular wavelength oscillates because of the interference effect [16], [17]. The blue-shift and then the red-shift trends in Fig. 7 can be attributed to the interference oscillation. Fig. 8 shows the optical intensity variations at $\lambda=500 \mathrm{~nm}$ versus the ITO, HTL, EML, and ETL thickness. The calibrated periods of the oscillatory behaviors are about $140,145,140$, and $144 \mathrm{~nm}$ in varying the ITO, HTL, EML, and ETL thicknesses, respectively. These periods roughly correspond to one-half of the wavelength in the OLED materials. We

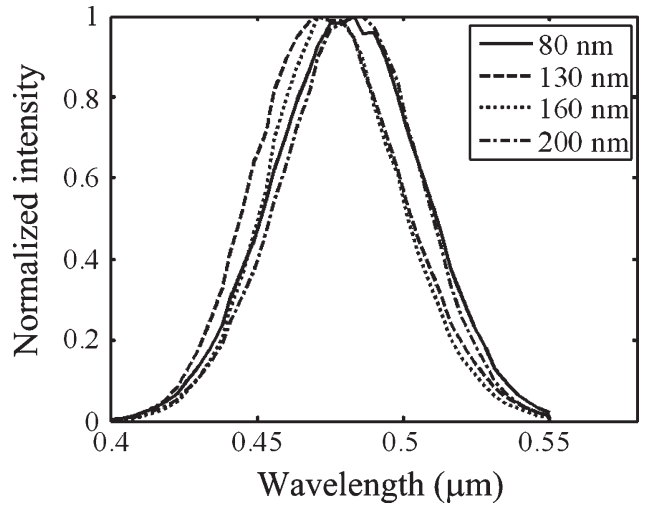

Fig. 7. Simulation results of the EL spectra at $\theta=0$ with various HTL thicknesses.

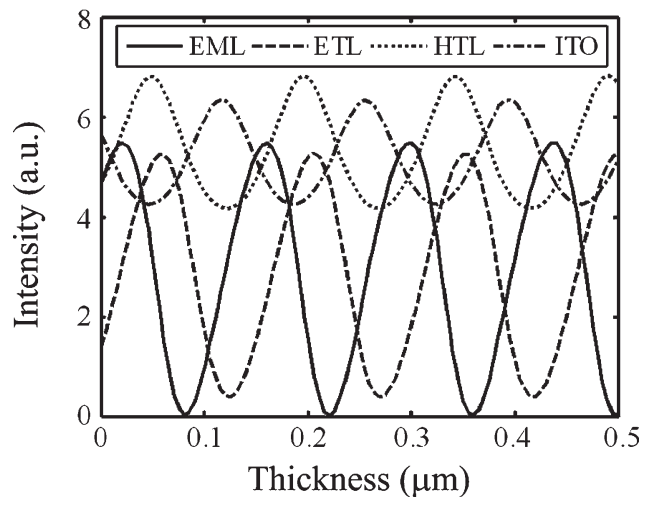

Fig. 8. Intensities at $\lambda=500 \mathrm{~nm}$ and $\theta=0$ as functions of the ITO, HTL, EML, and ETL thicknesses.

also note that the optical intensity oscillations in changing the EML and ETL thicknesses are stronger than those of changing the HTL and ITO thicknesses. In an OLED, the strongest single-face reflection occurs at the interface between the ETL and cathode layer. Therefore, the variation of the ETL thickness generates strong intensity oscillation. In addition, varying the EML or ETL thickness is equivalent to changing the optical path between the dipole source and the ETL/cathode interface. Such a change may strongly affect the radiation output intensity, as shown in Fig. 8.

Note that the real parts of the refractive indexes of the organic layers NPB and Alq3 are around 1.7 to 1.8 [16], [18]. In our simulations, the real parts of the refractive indexes of these dielectric materials were set to be constants within the whole visible range, as shown in Table I. Actually, the real part of the refractive index of an organic material is dispersive and follows the Cauchy model. Such dispersion behaviors can also be considered in our simulations. Fig. 9 shows the EL spectra at $\theta=0$ with various HTL thicknesses at $80,130,160$, and $200 \mathrm{~nm}$. The parameters used for calculations are the same as those in Fig. 7, except that the refractive indexes of NPB (for HTL) and Alq3 (for ETL) become dispersive now. ${ }^{1}$ One can see that the EL peaks are located at $483,479,479$, and $483 \mathrm{~nm}$ for the four HTL thicknesses, respectively. Such an

\footnotetext{
${ }^{1}$ From the Cauchy model, the refractive index of NPD is $\mathrm{n}=1.7118+$ $0.03366 / \lambda^{2}$ ( $\lambda$ in microns), and that of Alq3 is $n=1.6434+0.02533 / \lambda^{2}$ ( $\lambda$ in microns) from measurements.
} 


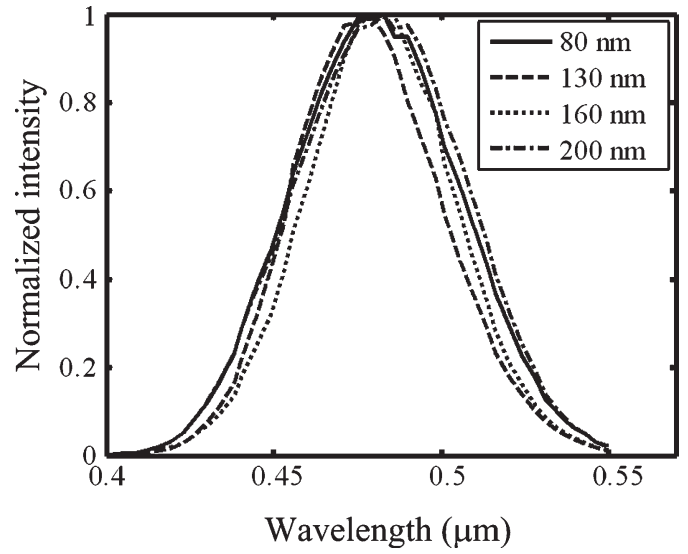

Fig. 9. Simulation results of the EL spectra at $\theta=0$ with various HTL thicknesses when the dispersion behaviors are considered.

oscillatory behavior, which is due to the optical interference effect, is similar to that in Fig. 7. The slight variation in the EL peak position results from the change of optical length through the dispersion. Therefore, the dispersion does not significantly affect the output characteristics of an OLED. Hence, in our simulations, the real parts of the refractive indexes of the organic layers are set to be constants for simplifying the calculations.

Within the visible range, the imaginary parts of the refractive indexes of NPB, ADN, and DPAVBi can be neglected due to their large band gaps [19]. The onset of the $\pi-\pi^{*}$ absorption band of Alq3 is located around $450 \mathrm{~nm}$. Its absorption increases with photon energy and reaches a peak at $390 \mathrm{~nm}$. However, because the primary emission spectra of OLEDs lie at longer wavelengths, as shown in Fig. 6, the absorption behavior of Alq3 should not significantly affect our simulation results. In addition, because the glass substrate is transparent and ITO is semitransparent, the absorption/gain dispersion behaviors of the used materials were neglected in our simulations. The negligence may explain the discrepancy between the simulation [Fig. 6(a)] and experimental [Fig. 6(b)] results at the shortwavelength tail. Nevertheless, the dispersive behaviors of those cathode metals [15] are important and were really taken into account in our simulations.

Other than the radiation into the free space, part of the dipole power is either trapped by the guided modes of the layered medium or absorbed by the device materials. Calculations of these powers for a dipole embedded in a dielectric slab have been carried out by Brueck [12]. However, the geometry of our problem is more complicated. In our device, we consider seven layers such that the boundary conditions at six interfaces must be matched. Analytical solutions seem impossible in our geometry. In the spectral domain of the Fourier-Bessel transform, only the numerical results of various expansion coefficients can be obtained in solving a matrix equation. Therefore, we could not take the advantage of evaluating the residues of poles in the spectral representation such that the guided power can be calculated. However, because we are interested in the radiation behaviors in the outmost air region, the use of the saddle-point approximation is a good choice. Because the feasible field computation in the air region represents one of the advantages of our numerical model, we can calculate the total radiated power in the air region and divide it by the total radiated power of a dipole in the free space to estimate the external quantum efficiency. It is found that the external quantum efficiency is around $17 \%$ for a vertical dipole when the optimal layer thickness is used in our simulation. Although it is difficult to estimate the external quantum efficiency of a horizontal dipole, it is believed to be higher when compared with that of a vertical dipole. Therefore, if $10^{5}$ random dipoles are averaged, the external quantum efficiency can be increased to about $25 \%$ under the optimized condition. This estimation is consistent with the value of a conventional OLED device [20].

\section{CONCLUSion}

Based on the rigorous electromagnetic wave theory, we have developed a numerical model for simulating the radiation characteristics of OLEDs. In particular, we proposed a new method for overcoming the numerical difficulty of including the effects of thick glass substrate in simulating the radiation properties of an OLED. The numerical results confirmed the importance of the effects of the thick glass substrate. The algorithms based on the numerical model were then used for evaluating the dependencies of OLED radiation characteristics on various parameters, including the thickness of different device layers and the metal type used for the cathode. With regard to the effect of EML thickness, it was found that the radiation spectral peak red shifted with increasing EML thickness. This trend is consistent with the experimental result.

\section{REFERENCES}

[1] C. W. Tang and S. A. VanSlyke, "Organic electroluminescent diodes," Appl. Phys. Lett., vol. 51, no. 12, pp. 913-915, Sep. 1987.

[2] C. W. Tang, S. A. VanSlyke, and C. H. Chen, "Electroluminescence of doped organic thin films," J. Appl. Phys., vol. 65, no. 9, pp. 3610-3616, May 1989.

[3] X. Jiang, P. Herguth, T. Sassa, and A. K-Y. Jen, "Efficient green polymer light-emitting diodes with microcavity effect in electroluminescence spectrum but constant quantum efficiency," J. Appl. Phys., vol. 96, no. 6, pp. 3553-3555, Sep. 2004.

[4] B. D. Chin, M. C. Suh, S. T. Lee, and H. K. Chung, "Improved blue light-emitting polymeric device by the tuning of drift mobility and charge balance," Appl. Phys. Lett., vol. 84, no. 10, pp. 1777-1779, Mar. 2004.

[5] K. B. Kahen, "Rigorous optical modeling of multilayer organic lightemitting diode devices," Appl. Phys. Lett., vol. 78, no. 12, pp. 1649-1651, Mar. 2001.

[6] J. Rostalski and D. Meissner, "Photocurrent spectroscopy for the investigation of charge carrier generation and transport mechanisms in organic p/n-junction solar cells," Sol. Energy Mater. Sol. Cells, vol. 63, no. 1, pp. 37-47, Jun. 2000

[7] P. Peumans, A. Yakimov, and S. R. Forrest, "Small molecular weight organic thin-film photodetectors and solar cells," J. Appl. Phys., vol. 93, no. 7, pp. 3693-3723, Apr. 2003.

[8] H. Riel, S. Karg, T. Beierlein, W. Rieß, and K. Neyts, "Tuning the emission characteristics of top-emitting organic light-emitting devices by means of a dielectric capping layer: An experimental and theoretical study," J. Appl. Phys., vol. 94, no. 8, pp. 5290-5296, Oct. 2003.

[9] C. C. Wu, C. L. Lin, P. Y. Hsieh, and H. H. Chiang, "Methodology for optimizing viewing characteristics of top-emitting organic light-emitting devices," Appl. Phys. Lett., vol. 84, no. 20, pp. 3966-3968, May 2004.

[10] L. M. Brekhovskikh, Waves in Layered Media. New York: Academic, 1960.

[11] J. R. Wait, Electromagnetic Waves in Stratified Media. Oxford, U.K.: Pergamon, 1962.

[12] S. R. J. Brueck, "Radiation from a dipole embedded in a dielectric slab," IEEE J. Sel. Topics Quantum Electron., vol. 6, no. 6, pp. 899-910, Nov./Dec. 2000. 
[13] A. Ishimaru, Electromagnetic Wave Propagation, Radiation, and Scattering. Englewood Cliffs, NJ: Prentice-Hall, 1991.

[14] G. Gu, D. Z. Garbuzov, P. E. Burrows, S. Venkdtesh, and S. R. Forrest, "High-external-quantum-efficiency organic light-emitting devices," Opt. Lett., vol. 22, no. 6, pp. 396-398, Mar. 1997.

[15] Handbook of Optical Constants of Solids, E. D. Palik, Ed. New York: Academic, 1985.

[16] Y. Fukuda, T. Watanabe, T. Wakimoto, S. Miyaguchi, and M. Tsuchida, "An organic LED display exhibiting pure RGB colors," Synth. Met., vol. 111/112, pp. 1-6, Sep. 2000.

[17] S. K. So, W. K. Choi, L. M. Leung, and K. Neyts, "Interference effects in bilayer organic light-emitting diodes," Appl. Phys. Lett., vol. 74, no. 14, pp. 1939-1941, Apr. 1999.

[18] S. Han, C. Huang, and Z. H. Lu, "Color tunable metal-cavity organic light-emitting diodes with fullerene layer," J. Appl. Phys., vol. 97, no. 9, p. 093102 , Apr. 2005.

[19] P. A. Hobson, J. A. E. Wasey, I. Sage, and W. L. Barnes, "The role of surface plasmons in organic light-emitting diodes," IEEE J. Sel. Topics Quantum Electron., vol. 8, no. 2, pp. 378-386, Mar./Apr. 2002.

[20] Y. R. Do, Y .C. Kim, Y. W. Song, and Y. H. Lee, "Enhanced light extraction efficiency from organic light emitting diodes by insertion of a twodimensional photonic crystal structure," J. Appl. Phys., vol. 96, no. 12, pp. 7629-7636, Dec. 2004.

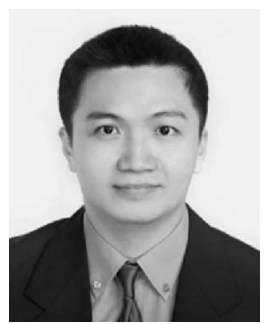

Hung-Chi Chen was born in Taiwan, R.O.C., in 1974. He received the B.S.E.E. degree from National Taiwan University of Science and Technology, Taipei, Taiwan, in 2000 and the M.S.E.E. degree from National Taiwan University, Taipei, in 2003.

$\mathrm{He}$ is currently a Full-Time Teaching Assistant with the electric experiment laboratory in the Department of Electrical Engineering, National Taiwan University.

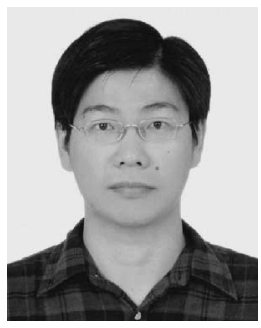

Jiun-Haw Lee (M'03) was born in Taipei, Taiwan, R.O.C., on August 20, 1972. He received the B.S.E.E., M.S.E.E., and Ph.D. degrees from National Taiwan University, Taipei, in 1994, 1995, and 2000, respectively, all in electrical engineering.

From 2000 to 2003, he was with the RiTdisplay Corporation as the Director. Since 2003, he has been with the Graduate Institute of Electro-optical Engineering and the Department of Electrical Engineering, National Taiwan University, as an Assistant Professor. His research interests include organic light emitting displays, display technologies, and solid-state lighting.
Chia-Chiang Shiau was born in Changhwa, Taiwan, R.O.C., in 1976. He received the B.S.E.E. degree from Tsing Hua University, Hsinchu, Taiwan, R.O.C., in 2000 and the M.S.E.E. degree from the National Taiwan University, Taipei, Taiwan, R.O.C., in 2005.

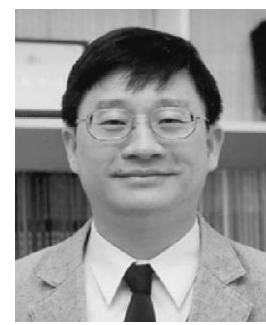

Chih-Chung Yang (S'81-M'83-SM'93) received the B.S. degree from the Department of Electrical Engineering, National Taiwan University, Taipei, Taiwan, R.O.C., and the M.S. and Ph.D. degrees in electrical engineering from the University of Illinois, Urbana-Champaign, in 1984.

He was a Faculty Member in the Department of Electrical Engineering, Pennsylvania State University, University Park, for nine years. In 1993, he then joined the Graduate Institute of Electro-Optical Engineering, National Taiwan University, as a Full Professor and has been serving as its Chairman since 2001. His research areas include the metal-organic chemical vapor deposition growth of nitride compounds, nanostructure materials, white light light-emitting diodes, photonic crystals, surface plasma waves, and biophotonics.

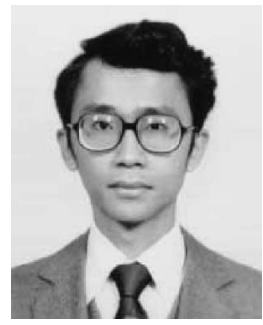

Yean-Woei Kiang ( $\left.\mathrm{S}^{\prime} 81-\mathrm{M}^{\prime} 84\right)$ was born in Panchiao, Taiwan, R.O.C., in 1954. He received the B.S.E.E., M.S.E.E., and Ph.D. degrees from the National Taiwan University, Taipei, Taiwan, R.O.C., in 1977, 1979, and 1984, respectively, all in electrical engineering.

In 1979, he joined the faculty of the Department of Electrical Engineering, National Taiwan University, where he is currently a Professor. He is also with the Graduate Institute of Communication Engineering. His research interests include wave propagation, scattering, inverse scattering, and optoelectronics. 